\title{
Prevalence of perennial severe allergic asthma in Italy and effectiveness of omalizumab in its management: PROXIMA - an observational, 2 phase, patient reported outcomes study
}

Giorgio Walter Canonica ${ }^{1}$, Marta Bartezaghi², Raffaele Marino ${ }^{2}$ and Laura Rigoni ${ }^{2 *}$

\begin{abstract}
Background: We designed the PROXIMA study (Patient-Reported Outcomes and Xolair In the Management of Asthma) to determine the proportion of patients with severe asthma sensitive to perennial allergens, and to evaluate asthma control and treatment adherence up to 12 months in patients treated with omalizumab in Italian population. In addition, an ancillary study was designed to explore protein biomarkers and characterize them in relation to severe allergic asthma and treatment effects by proteomic approach.
\end{abstract}

Methods: PROXIMA is an observational, multicenter, cross-sectional and prospective cohort study conducted at 25 centers in Italy, in outpatient settings. The study consists of two phases: 1) a cross-sectional phase plans to enroll 600 patients with severe allergic asthma, in step 4 therapy as per GINA guidelines, aged $\geq 18$ years, needing a step up in therapy, and 2) a longitudinal phase on patients who will start omalizumab add-on therapy per clinician's judgment at baseline visit (approximately 180-240 patients). The primary variable of the cross-sectional phase is the proportion of patients with severe asthma presenting with perennial form of allergy (skin prick test or in vitro test). The primary variable of longitudinal phase is proportion of patients who achieve disease control (assessed by Asthma Control Questionnaire [ACQ]) with omalizumab at 6 months, and maintain it at 12 months. Secondary variables are patient compliance to omalizumab, patient-reported perception of cognitive and emotional impact of the illness, assessed by Brief Illness Perception Questionnaire (Brief IPQ) and the health related quality of life evaluated by the EuroQoL 5D-3 L (EQ-5D-3 L). Safety endpoints will be recorded during the course of the study. Patients participating in the longitudinal phase will be enrolled for ancillary study if they provide additional informed consent. Protein species in complex mixtures will be identified using innovative MudPIT (Multidimensional Protein Identification Technology) method.

Conclusions: The results of this observational study will provide estimate of patient population allergic to perennial allergens in Italy and information on patient-reported outcomes with omalizumab therapy in a real-world setting. The exploratory proteomic analysis on asthma biomarkers could eventually provide new data to identify responder patients to anti lgE therapy.

Keywords: Omalizumab, IgE, Severe allergic asthma, Perennial, Asthma control

\footnotetext{
* Correspondence: laurarigoni@novartis.com

${ }^{2}$ Novartis Farma SpA, Largo U. Boccioni 1, Origgio (VA) 21040, Italy

Full list of author information is available at the end of the article
} 


\section{Background}

Asthma is a highly prevalent respiratory disorder characterized by chronic inflammation and hyperresponsiveness of airways, and variable airflow obstruction, often reversible [1]. More than 300 million people are affected by asthma, worldwide [1,2]. Of this population, about $10 \%$ is estimated to have severe asthma accounting for significant burden of morbidity and mortality [3,4]. In Italian population (GEIRD Study), the median prevalence of asthma was reported to be $6.6 \%$, ranging from $4.5 \%-8.5 \%$, recording a $35 \%$ increase in the last 2 decades [5]. Clinically, many different forms or phenotypes of asthma have been recognized; however, allergic asthma affects a substantial proportion of patients with asthma [2]. Atopy and allergic mechanisms have been implicated for asthma in $50-80 \%$ of the patients and in approximately $50 \%$ of patients with severe asthma [6-8]. In Italy, allergic asthma affects $>60 \%$ patients with asthma [5].

In patients with allergic asthma, who are already sensitized to a specific environmental allergen, exposure to same allergen acts as powerful trigger for asthmatic response, which is mast-cell driven in the early phase and inflammatory-cell driven in late phase $[1,8]$. Markedly increased levels of IgE antibodies are found in individuals with allergic asthma. IgE antibodies cause chronic airway inflammation via high-affinity (FceRI) or lowaffinity (FcERII) IgE receptors present on effector cells such as mast cells, and basophils $[1,8]$. Antigen induced cross-bridging of IgE-FcERI cell surface complexes, triggering degranulation of effector cells, gives rise to various symptoms of allergy. The distinction between perennial and seasonal allergic asthma are often made on the basis of the seasonality of sensitizing allergens (seasonal and perennial) and the detection IgE specific to allergen [8]. However, distinction between seasonal and perennial allergic asthma based on seasonality of allergens has been a topic for discussion among clinicians because of certain overlapping characteristics of allergens. Recently, it was proposed to replace the terms seasonal and perennial with the 'intermittent' and 'persistent' - disease or -exposure to allergens. The concept was intended to introduce change in the treatment approach for allergic diseases taking into account the overlap between the treatment of seasonal and perennial allergies. The new definition was proposed because the concept of seasonality could not be applied to some of the perennial allergens (occupational, mite and pet allergens) and some seasonal environmental allergens (pollen) might be considered as perennial owing changes in climate. Moreover, the majority of patients are usually sensitized with multiple allergens (polysensitization) with occupational allergens, mite and pet allergens, and presence of cross-reactivity and pan-allergens [9].
Omalizumab, a recombinant monoclonal antibody (IgE antibody), reduces free IgE levels by binding to the Fc domain of the circulating IgE and thereby inhibiting IgE binding to FceRI receptors. In addition omalizumab also blocks the IgE-dependent uptake of allergens by mature myeloid dendritic cells, thereby affecting the chronic allergic response $[7,10]$. Currently, omalizumab is the recommended treatment of severe allergic asthma in patients not responding to Step 4 of the GINA treatment approach, and need therapeutic step up to Step 5 [11]. In the European Union, omalizumab is approved as add-on therapy in adults, adolescents, and children ( 6 to $<12$ years of age) with severe persistent allergic asthma inadequately-controlled (reduced lung function, frequent daytime symptoms or night-time awakenings, and multiple documented severe asthma exacerbations), despite daily high-dose inhaled corticosteroids (ICS), plus a long-acting inhaled beta2-agonist (LABA) [12].

Medical literature lacks information on the proportion of patients with severe asthma presenting with the perennial form of allergy in Italy. Moreover, data on the effectiveness of omalizumab as add-on therapy for patients with severe persistent allergic asthma in the Italian population is limited.

\section{Primary objectives}

The PROXIMA (Patient Reported Outcomes and Xolair In the Management of Asthma) study was designed to estimate the prevalence of perennial allergy in adult patients with severe allergic asthma in the Italian population and to evaluate the proportion of patients in this demographics who achieve disease control at 6 months and maintain it at 12 months in a sample of patients treated with omalizumab.

\section{Methods}

\section{Study design and patient population}

PROXIMA is an observational, multicenter, crosssectional and prospective cohort study conducted at 25 centers in Italy, in outpatient settings (Hospitals and University centers specialized in the treatment of asthma). The study comprises of 2 phases, a crosssectional and a longitudinal phase (12 months). Patients entered in the longitudinal phase will be treated with omalizumab as per "scheda tecnica" [12] (Figure 1).

In the cross-sectional phase, about 600 patients aged $\geq 18$ years, diagnosed with severe allergic asthma currently in Step 4 (as defined by GINA international guidelines) and need a step up in therapy will be enrolled. About 30\%-40\% (180-240 patients with perennial allergic asthma) are estimated to enter the longitudinal phase, start therapy with omalizumab, followed up for 12 months. Omalizumab as treatment choice will be based on clinician's judgment and local treatment guidelines. As the 


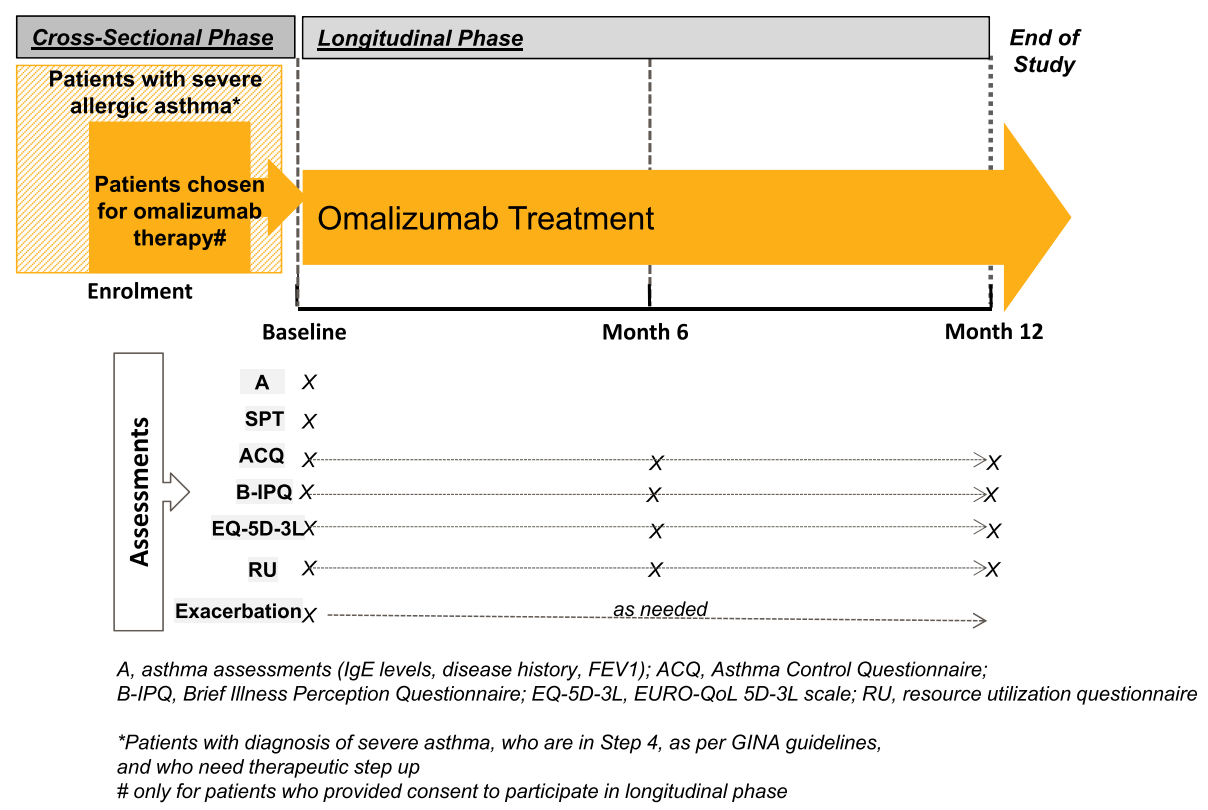

Figure 1 Study design and assessments.

study will involve questionnaire based assessments, patient unable to complete the patient questionnaires will not be enrolled for the study. Patients participating in the longitudinal phase will have the option to participate to an ancillary study (The PROXIMA sub-study: exploratory proteomic analysis on asthma biomarkers).

This study was designed and shall be implemented and reported in accordance with the Guidelines for Good Pharmacoepidemiology Practices (GPP) of the International Society for Pharmacoepidemiology (ISPE 2008), the STROBE (Strengthening the Reporting of Observational Studies in Epidemiology) guidelines, the ethical principles laid down in the Declaration of Helsinki, and the AIFA (Agenzia Italiana del Farmaco) guideline for the classification and management of observational studies on drugs [13-15].

Eligible patients may be enrolled only after written informed consent. The study documents including protocol and informed consent forms were approved by the ethics committees and institutional review boards of all participating centers.

\section{Variables}

The two primary variables of the study are the proportion of patients with severe allergic asthma having allergy to a perennial form of aeroallergens (at baseline cross-sectional phase) and the proportion of patients with asthma control at Months 6 and 12 (in longitudinal phase) with omalizumab therapy. Secondary variables in the cross-sectional phase are the level of asthma control, patient disease perception at baseline in all patients (whole sample) and in patients with perennial allergy compared with seasonal allergy, quality of life and healthcare resource utilization. In the longitudinal phase secondary variables are, 1) proportion of patients with at least 1 episode of asthma exacerbation during the 12 months study period; 2) patient compliance to omalizumab at 6 and 12 months; 3 ) persistence with omalizumab treatment for 12 month; and 4) quality of life and healthcare resource utilization at months 6 and 12 .

\section{Assessments}

Patients with perennial form of allergy will be identified by clinicians on the basis of the results of skin prick test or in vitro tests for the reactivity to a perennial aeroallergen. Asthma disease control will be assessed by the Asthma Control Questionnaire (ACQ) using a 7-point scale, where 0 indicates well-controlled and 6 signify extremely poorly controlled asthma [16]. A patient will be defined (as per protocol) as controlled if the ACQ total score will be between 0 and 4 .

Brief Illness Perception Questionnaire (Brief IPQ) and EuroQoL 5D-3 L (EQ-5D-3 L) scale will be used to assess patient disease perception and quality of life, respectively. Brief IPQ is a 9-item questionnaire designed to rapidly assess cognitive and emotional representations of illness [17]. All of the questionnaire items (except the causal question, item 9) are rated using a 0 to-10 response scale; five of the items assess cognitive illness representations, two of the items assess emotional representations and one item assesses illness comprehensibility. Assessment of the causal representation is by an 
open response item, which asks patients to list the three most important causal factors in their illness. EQ-5D$3 \mathrm{~L}$ scale consists of two sections, EQ-5D-descriptive system and the EQ-visual analogue scale (EQ-VAS) $[18,19]$. The EQ-5D-3 L descriptive system assesses 5 dimensions: mobility, self-care, usual activities, pain/ discomfort and anxiety/depression. Each dimension has 3 levels: no problems, some problems, extreme problems. EQ-VAS is used as a quantitative measure of health as judged by the individual respondents on a visual analogue scale from 0 to 100 , where 0 the "worst imaginable health state" and 100 is the "best imaginable health state".

Patient compliance will be assessed as the ratio between the number of injections of omalizumab administered during the observational period (month 6 and 12) over the total number of planned injections. Omalizumab persistence during the 12 months will be assessed by Kaplan-Meier survival curve analysis, where the event of interest will be the treatment discontinuation.

The consumption of healthcare resources will be assessed by using a dedicated questionnaire collecting information on the number of hospitalizations, number of emergency department visits, number of outpatient visits and number of laboratory and diagnostic tests.

Asthma exacerbations, serum IgE levels and forced expiratory volume in $1 \mathrm{~s}\left(\mathrm{FEV}_{1}\right)$ percent (at baseline) will be measured by using standard clinical and laboratory assessment methods [20]. Safety assessments consist of clinical examination, monitoring and recording all adverse events and serious adverse events. All events will be reported to local Health Authorities according to Italian regulatory requirements and recorded in the Novartis safety database.

\section{Statistical considerations}

The primary variable in the cross-sectional phase the proportion of patients with a perennial form of aeroallergens will be calculated as the ratio of the number of patients with a perennial form of aeroallergens to the total number of evaluable patients with a diagnosis of severe allergic asthma at baseline.

The primary variable in the longitudinal phase, the proportion of controlled patients based on ACQ both at 6 months and at 12 months, will be calculated as the ratio between the patients who are controlled and the total number of evaluable patients treated with omalizumab. Both of the primary variables will be presented with 95\% confidence limit. The proportion of patients who change status from controlled to un-controlled and vice-versa between 6 and 12 months will also be presented.

With respect to the longitudinal phase, descriptive statistics for each time point as well as changes versus baseline will be provided for the ACQ total score, brief
IPQ and EQ-5D-3 L. The patient compliance with omalizumab use will be evaluated, as the ratio between the numbers of administered injections during the observational period over the total number of planned injections, at 6 and 12 months after treatment start.

\section{Sample size estimations}

The sample size was determined on the basis of feasibility criteria. According to the volume of patients managed by the centers involved in this study, it is reasonable to suppose the inclusion of 600 eligible outpatients are adequate to arrive at sufficient sample size for the study. Considering that $5 \%$ patients may not be available for analysis, because of missing data or inclusion-exclusion criteria violations, a total of 570 evaluable patients, allows a precision of estimate between $2.5 \%$ and $3.9 \%$ of the proportion of patients with a perennial form of aeroallergens. In particular the two sided 95\% confidence interval will be $7.5 \%-12.5 \%$ for the $10 \%$ expected proportion of patients and $31.1 \%-38.9 \%$ for the $35 \%$ expected proportion, based on the results of ENFUMOSA study [3]. The proportion of patients starting omalizumab at baseline is expected to range between $30 \%-40 \%$. Therefore, the sample size of patients to be included in the longitudinal phase should range from 180-240 patients. Assuming a 12 months drop-out rate (including non-evaluable patients) of about $15 \%, 153-204$ patients will be evaluable for the primary endpoint in the longitudinal phase. Moreover, based on the response of the omalizumab treatment as assessed by using global evaluation of treatment effectiveness (GETE) tool, it was appropriate to assume that the proportion of patients with disease control after 12 months will range from 50\%-70\%. A sample of 153 and 204 evaluable patients allows a precision of estimate between $7.9 \%-7.3 \%$ and $6.9 \%-6.3 \%$, for the proportion of patients who achieve the disease control at month 6 and maintains it at month 12 [21,22].

\section{The PROXIMA ancillary-study: exploratory proteomic analysis on asthma biomarker Rationale}

Asthma is a complex respiratory disorder known to have multiple causes, phenotypes, and treatment responses. However, this complexity provides an opportunity for personalized and targeted therapy by characterizing specific biomarkers aiding to early diagnosis, assessing therapeutic responses and even validation for new drug targets [23]. Recent advances in mass spectrometrybased proteomic approaches may facilitate identification and characterization of new biomarkers in asthma and subsequently improve understanding of their clinical significance $[24,25]$.

Currently, biomarkers that have known diagnostic and clinical role in asthma are poorly characterized and there 
are no proteomic-based studies to describe inflammatory biomarkers during and after the treatment of severe asthma.

\section{Objectives}

This ancillary study was designed to explore protein biomarkers in patients with severe allergic asthma treated with omalizumab and characterize potential biomarkers in relation to the achievement and maintenance of disease control. In addition, the study aims to identify proteotypical peptides useful for the development of diagnostic methods.

\section{Study design and patient population}

The PROXIMA ancillary study is planned to be conducted at 8 centers in Italy with the aim of characterizing biomarkers related to severe allergic asthma by a proteomic approach. The patients who would participate in the longitudinal phase of PROXIMA study and provide additional informed consent specific to the ancillary study will be enrolled.

\section{Sample collections and investigations}

Three samples for blood and urine will be collected from eligible patients at baseline, 6 and 12 months of the PROXIMA main study. The samples will be collected, stored and shipped, as per instructions defined by the central laboratory, to conduct the proteomic analysis at Internal Medicine Department at Respiratory Diseases Clinic, Hospital San Martino, University of Genoa.

A gel-free system and mass spectrometry will be used to explore the proteins biomarkers. Protein species in complex mixtures will be identified using innovative MudPIT (Multidimensional Protein Identification Technology) method [26]. The MudPIT method consists of a twodimensional chromatography coupled to the mass spectrometry in tandem (2 DC-MS/MS) that allows the identification of thousands of proteins simultaneously from single complex sample [27].

\section{Statistical analysis}

There was no formal statistical hypothesis was set for the study. Descriptive analyses will be performed. The list and frequency of all proteins identified in the total sample of patients will be provided at baseline, 6 and 12 months of omalizumab treatment. In order to assess whether asthma protein biomarkers are related to the achievement and maintenance of disease control, the frequency of all identified proteins will be stratified by responders and non-responders (as defined in the PROXIMA main study). Since a large number of possible proteins are expected to be observed, Bonferroni's correction will be applied. The $95 \%$ confidence interval will be calculated for both objectives.

\section{Discussion and conclusions}

The PROXIMA observational study is designed to provide estimates on the number of patients with asthma who are allergic to perennial allergens and to assess the effectiveness of omalizumab in patients with severe allergic asthma in the Italian population for the first time. In various clinical studies, omalizumab has been shown to improve asthma control and reduce exacerbations in patients with inadequately-controlled severe allergic asthma [4]. Current study is planned to be conducted in a real-world setting in Italy and the result will further confirm the control of asthma achieved and maintained by omalizumab therapy in this population. The exploratory proteomic analysis on asthma biomarkers could identify proteins of interest in patients with asthma, and link them to the patients' level of response to the disease and treatment. The new data that will help to identify and characterize the best responders to omalizumab treatment will be a step towards personalized medicine.

\section{Abbreviations \\ 2 DC-MS/MS: Two-dimensional chromatography coupled to the mass spectrometry in tandem; ACQ: Asthma Control Questionnaire; AIFA: Agenzia Italiana del Farmaco; Brief IPQ: Brief Illness Perception Questionnaire; EQ VAS: EQ-visual analogue scale; EQ-5D-3 L: EuroQoL 5D-3 L; FEV 1 : Forced expiratory volume in $1 \mathrm{~s}$; GETE: Global evaluation of treatment effectiveness; GINA: Global Initiative for Asthma; GPP: Good Pharmacoepidemiology Practices; ICS: Inhaled corticosteroids; IgE: Immunoglobulins E; ISPE: International Society for Pharmacoepidemiology; LABA: Long-acting inhaled beta2-agonist; MudPIT: Multidimensional Protein Identification Technology; PROXIMA: Patient Reported Outcomes and Xolair In the Management of Asthma; STROBE: Strengthening the Reporting of Observational Studies in Epidemiology.}

\section{Competing interests}

Giorgio Walter Canonica, received honoraria, speaker, or consulting fees from Novartis.

Marta Bartezaghi, Raffaele Marino, and Laura Rigoni are employees of Novartis Farma SpA.

\section{Authors' contributions}

GWC, RM, and LR conceived the study, participated in its design and analysis plan. BM participated in the study design and analysis plan. All authors read and approved the final manuscript.

\section{Acknowledgements}

We thank Arun Kumar Singh who provided medical writing support on behalf of Novartis Healthcare Private Limited, Hyderabad, India.

\section{Funding}

The study and this work were sponsored by Novartis Farma S.p.A., Origgio (VA), Italy.

\section{Author details \\ 'DIMI, IRCCS AOU San Martino, University of Genoa, Genoa, Italy. ${ }^{2}$ Novartis Farma SpA, Largo U. Boccioni 1, Origgio (VA) 21040, Italy.}

Received: 3 March 2015 Accepted: 8 April 2015

Published online: 07 July 2015

\footnotetext{
References

1. Mukherjee $A B$, Zhang Z. Allergic asthma: influence of genetic and environmental factor. J Biol Chem. 2011;286(38):32883-9.
} 
2. Shifren A, Witt C, Christie C, Castro M: Mechanisms of Remodeling in Asthmatic Airways. J Allergy. Volume 2012 (2012). http://dx.doi.org/ 10.1155/2012/316049

3. The ENFUMOSA Study Group. The ENFUMOSA cross-sectional European multicentre study of the clinical phenotype of chronic severe asthma. Eur Resp J. 2003;22(3):470-7.

4. McKeage K. Omalizumab: a review of its Use in patients with severe persistent allergic asthma. Drugs. 2013;73:1197-212.

5. de Marco R, Cappa V, Accordini S, Rava M, Antonicelli L, Bortolami O, et al Trends in the prevalence of asthma and allergic rhinitis in Italy between 1991 and 2010. Eur Respir J. 2012;39:883-92.

6. Price D. The use of omalizumab in asthma. Prim Care Respir J. 2008;17(2):62-72.

7. D'Amato G, Stanziola A, Sanduzzi A, Liccardi G, Salzillo A, Vitale C, et al. Treating severe allergic asthma with anti-lgE monoclonal antibody (omalizumab): a review. Multidisciplinary Respir Med. 2014;9:23.

8. Holgate ST. Stratified approaches to the treatment of asthma. Br J Clin Pharmacol. 2012;76:277-91

9. Definition of Allergens 2013. Is there a need for Seasonal \& Perennial? Available at URL: http://www.worldallergy.org/UserFiles/file/ GWCMANIFESTOALLERGENSCHICAGO_FullPage.pdf, accessed on 17 Dec, 2014

10. Schroeder JT, Bieneman AP, Chichester KL, Hamilton RG, Xiao H, Saini SS, et al. Decreases in human dendritic cell-dependent $T(H)$ 2-like responses after acute in vivo IgE neutralization. J Allergy Clin Immunol. 2010;125:896-901.

11. Global Strategy for Asthma Management and Prevention, Global Initiative for Asthma (GINA) 2014. Available at URL: http://www.ginasthma.org, accessed on Oct 27, 2014

12. European Medicines Agency. Xolair powder and solvent for solution for injection: summary of product characteristics; 2012. http://www.ema.europa.eu/ docs/en_GB/document_library/EPAR_-_Product_Information/human/000606/ WC500057298.pdf (Accessed 23 Sep 2014).

13. Guidelines for good pharmacoepidemiology practices (GPP). Pharmacoepidemiology and drug safety 2008; 17: 200-208. (available at URL: http://www.pharmacoepi.org/pub/1c2a23af-2354d714-516a-7175549e3a88, accessed on 26 Sep, 2014).

14. von Elm E, Altman DG, Egger M, Pocock SJ, Gøtzsche PC, Vandenbroucke JP. STROBE initiative: strengthening the reporting of observational studies in epidemiology. J Clin Epidemiol. 2008;61(4):344-9.

15. AIFA Guideline for the classification and management of observational studies on drugs (Determination March 20, 2008). Available at URL: http://www.agenziafarmaco.com/en, accessed on January 12, 2015.

16. Juniper EF, Bousquet J, Abetz L, Bateman ED. Identifying 'well-controlled' and 'not well-controlled' asthma using the asthma control questionnaire. Respir Med. 2006;100:616-21.

17. Broadbent E, Petrie KJ, Main J, Weinman J. The brief illness perception questionnaire. J of Psychosom Res. 2006;60:631-7.

18. The EuroQoL Group. "EuroQol: a new facility for the measurement of health-related quality of life". Health Policy. 1990;16:199-208.

19. Brazier J, Jones $N$, Kind P. Testing the validity of the euroqol and comparing it with the SF-36 health survey questionnaire. Qual Life Res. 1993;2:169-80

20. Reddel HK, Taylor DR, Bateman ED, Boulet LP, Boushey HA, Busse WW, et al. An official american thoracic society/european respiratory society statement: asthma control and exacerbations: standardizing endpoints for clinical asthma trials and clinical practice. Am J Respir Crit Care Med. 2009;1(180):59-99.

21. Pelaia G, Gallelli L, Romeo P, Renda T, Busceti MT, Proietto A, et al. Omalizumab decreases exacerbation frequency, oral intake of corticosteroids and peripheral blood eosinophils in atopic patients with uncontrolled asthma. Int J Clin Pharmacol Ther. 2011;49(12):713-21.

22. Vennera Mdel C, Pérez De Llano L, Bardagí S, Ausin P, Sanjuas C, González $\mathrm{H}$, et al. Spanish registry: omalizumab therapy in severe asthma: experience from the spanish registry-some new approache. J Asthma. 2012:49:416-22.

23. Holgate ST. Asthma: a simple concept but in reality a complex disease. Eur J Clin Invest. 2011:41:1339-52.

24. Rossi R, De Palma A, Benazzi L, Riccio AM, Canonica GW, Mauri P. Biomarker discovery in asthma and COPD by proteomic approaches. Proteomics Clin Appl. 2014;8:901-15.
25. Mauri P, Riccio AM, Rossi R, Di Silvestre D, Benazzi L, De Ferrari L, et al. Proteomics of bronchial biopsies: Galectin-3 as a predictive biomarker of airway remodelling modulation in omalizumab-treated severe asthma patients. Immunol Lett. 2014;162:2-10.

26. Schirmer EC, Yates 3rd JR, Gerace L. MudPIT: a powerful proteomics tool for discovery. Discov Med. 2003;3(18):38-9.

27. Link AJ, Eng J, Schieltz DM, Carmack E, Mize GJ, Morris DR, et al. Direct analysis of protein complexes using mass spectrometry. Nat Biotechnol. 1999;17:676-82.

\section{Submit your next manuscript to BioMed Central and take full advantage of:}

- Convenient online submission

- Thorough peer review

- No space constraints or color figure charges

- Immediate publication on acceptance

- Inclusion in PubMed, CAS, Scopus and Google Scholar

- Research which is freely available for redistribution 